\title{
Dispositif expérimental pour étudier l'encrassement des échangeurs de chaleur alimentaires
}

\author{
par \\ M. ROIGNANT, G. DAUFIN et Françoise MICHEL*
}

\section{Rés u mé}

On évalue les caractéristiques thermiques et hydrodynamiques d'un dispositif expérimental destiné à l'étude au laboratoire de l'encrassement des échangeurs de chaleur. Le choix de dimensions convenables pour le préchauffeur assure la maîtrise et la connaissance du profil thermique et du niveau de température à l'entrée de la maquette d'encrassement. Dans celle-ci, des conditions thermiques et hydrodynamiques homogènes et quasi-constantes dans le temps peuvent être fixées indépendamment les unes des autres et varier dans de larges plages. On peut ainsi en étudier l'influence sur l'encrassement. L'aire réduite (quelques $\mathrm{cm}^{2}$ ) et la planéité de la surface d'étude permettent en outre d'utiliser des techniques physico-chimiques d'investigation des phases initiales de l'encrassement.

Titre abrégé :

Echangeur de chaleur expérimental pour l'encrassement. Mots clés:

Encrassement - Echangeur - Thermique - Hydrodynamique - Lait - Lactosérum.

\section{S u m m a ry}

Thermic and hydrodynamic characteristics of an experimental laboratory device for studying heat exchanger fouling have been evaluated. Choice of convenient sizes for the preheater ensures mastery and knowledge of thermal profile and temperature level at the product

* Institut National de la Recherche Agronomique, Laboratoire de Recherches de Technologie Laitière, 65, rue de Saint-Brieuc - 35042 Rennes cedex. 
inlet of the fouling simulating scale model. In the model, homogeneous and almost constant thermal and hydrodynamic conditions during the time can be fixed independently and vary within large area. So, their influence on fouling is allowed to be studied. Results of some tests carried out with skim milk and sweet whey are given; they confirm the well known influence of contact time, temperature and temperature difference (with heating or cooling fluid) on fouling measured by weighing mass of deposit. Furthermore, the reduced area (some $\mathrm{cm}^{2}$ ) and flatness of surface to be studied allow physicochemical techniques for investigating initial phases of fouling to be used.

Key words:

Fouling - Heat exchanger - Thermic - Hydrodynamics - Milk - Whey.

\section{INTRODUCTION}

$\mathrm{Au}$ cours de leur transformation, les produits alimentaires subissent pratiquement toujours un traitement thermique dont le principe repose le plus souvent sur un transfert de chaleur au travers d'interfaces liquide-solide (cas des échangeurs de chaleur à chauffage indirect tubulaire, à plaques, spirales, à surface raclée, ...).

Ces interfaces sont le lieu privilégié de réactions entre le matériau solide et les constituants du produit traité, ou entre les constituants eux-mêmes. Le résultat global est souvent un encrassement de la surface d'échange qui diminue les performances du matériel en opposant une résistance supplémentaire au transfert de chaleur (Burton, 1968) et en augmentant les pertes de charges (Lalande et al., 1981). En outre, il rend souvent plus difficiles et plus longues les opérations de nettoyage. Tous ces facteurs concourent à augmenter le coût de fonctionnement de l'installation.

Ces inconvénients ont justifié les études menées depuis de nombreuses années sur l'encrassement et sur les moyens de le limiter. Les revues bibliographiques de Burton (1968) et de Lund et Bixby (1975) font le point complet de ces travaux dans le domaine laitier où l'influence de divers paramètres relatifs à la matière première lait, ou aux conditions de l'échange thermique a été mise en évidence.

Cependant, les études sur un matériel industriel (comme celles de Lyster (1965) sur un échangeur à plaques pour traitement à ultra haute température, de Rota et Bottazzi (1978), de Corrieu et Lalande (1978) et de Lalande et al. (1981)) ou même pilote, s'avèrent difficiles : possibilités limitées de variations des conditions de traitement, variabilité des caractéristiques des produits traités, nécessité de disposer de quantités importantes de matière première, répartition hétérogène de l'encrassement, lourdeur de manipulation du matériel et difficultés d'interprétation de mesures souvent globales. Des études ont été également menées sur des matériels de laboratoire (Burton, 1961, 
1965 ; Bixby, 1974 ; Lund et Bixby, 1975 ; Thom, 1975 ; Ling et Lund, 1978 ; Meyer et al., 1981), dans des conditions expérimentales bien connues mais avec soit une absence de circulation, soit une recirculation du fluide encrassant.

D'une façon générale, l'ensemble des résultats acquis jusqu'à ces dernières années portait sur l'encrassement macroscopique. Or, les travaux exposés lors d'une manifestation sur le thème " Fundamentals and applications of surface phenomena associated with fouling and cleaning in food processing " (Suède, 1981), et parmi eux la revue bibliographique de Lund et Sandu (1981), soulignent que seule l'acquisition de connaissances nouvelles sur les premières phases de l'encrassement paraît désormais en mesure de contribuer à la lutte contre celui-ci. Pour ce faire, la mise en œuvre de méthodes de caractérisation des surfaces et d'études des phénomènes se produisant à l'interface solide-liquide est nécessaire (Ivarsson et al., 1981 ; Hegg et Larsson, 1981 ; Baier, 1981). Très fréquemment, ces méthodes ne s'appliquent qu'à des surfaces planes.

Ces différents éléments nous ont conduits à réaliser un matériel de traitement thermique de laboratoire qui devait répondre aux exigences suivantes :

- surface d'étude plane permettant de caractériser la surface du matériau solide étudié à l'état nu ou encrassé et l'interface solideliquide ;

- maîtrise des conditions thermiques et hydrodynamiques de travail, y compris les conditions de préchauffage du fluide encrassant, dont on connaît l'influence sur les phénomènes d'encrassement (Lyster, 1965 ; Gordon et al., 1968 ; Burton, 1968 ; Lund et Bixby, 1975 ; Rota et Bottazzi, 1978 ; Ling et Lund, 1978 ; Lalande et al,, 1981).

L'objectif principal de cet article est de présenter ce matériel et les résultats des essais entrepris pour le caractériser aux plans thermique et hydrodynamique. Ainsi, nous nous intéresserons successivement aux profils de température assurés dans le préchauffeur, aux échanges de chaleur dans la cellule d'encrassement (maquette) et aux caractéristiques de l'écoulement des fluides dans cette dernière. Enfin, quelques essais d'encrassement mettant en ouvre du lait et du lactosérum seront décrits, dont les résultats seront discutés en relation avec la validité du matériel pour l'étude de l'encrassement. Des comparaisons seront effectuées avec les résultats obtenus dans un échangeur à plaques industriel (prototype).

\section{MATERIEL ET METHODES}

\subsection{Maquette et son environnement}

Le matériel d'étude comportait une pompe de circulation du produit à étudier, un préchauffeur et un échangeur expérimental. 
La circulation du fluide dans le préchauffeur et l'échangeur était assurée par une pompe volumétrique à engrenages dont la caractéristique principale est un débit réglable de 0 à $2001 . h^{-1}$ à 6 bars de contrepression sur de l'eau (Bioblock : tête de pompe 86916 + système d'entraînement MC 31422 + aimant d'entraînement 31422). Le débit était mesuré par un débitmètre à flotteur (Brooks distribué par Prolabo). Les variations de débit au cours d'un essai n'excédaient pas $1 \%$ de la valeur nominale moyenne du débit.

Afin de porter le produit encrassant à sa température d'étude, nous utilisions un préchauffeur tubulaire. Celui-ci était constitué d'un tube en acier inoxydable (diamètre intérieur : $7 \times 10^{-3} \mathrm{~m}$ ) plongeant dans un bain-marie de 15 litres thermostaté à $\pm 0,1^{\circ} \mathrm{C}$.

Dans l'échangeur expérimental - maquette - (Fig. 1a et Fig. 1b) la surface d'échange à étudier était un échantillon en acier inoxydable du type $18 \%$ chrome $-10 \%$ nickel, de dimensions utiles $20 \times 40 \mathrm{~mm}$ et de $1,5 \mathrm{~mm}$ d'épaisseur.

Le long de l'une des faces circulait le produit encrassant dans un canal de section rectangulaire dont l'épaisseur pouvait varier de 0,1 à $4 \mathrm{~mm} \pm 10^{-2} \mathrm{~mm}$ grâce à l'utilisation d'un joint de polytétrafluoréthylène. Ceci permettait de faire varier les conditions d'écoulement du fluide encrassant.

De l'eau circulait le long de l'autre face à co ou contre courant et à débit connu $\left(200 \pm 10 \quad 1 . \mathrm{h}^{-1}\right)$ dans un canal de dimension $20 \times 40 \times 3 \mathrm{~mm}$. Les conditions d'échange de chaleur étaient définies par les températures de chacun des fluides (l'écart de température $\Delta t$ en découle évidemment). Ces deux températures étaient indépendantes l'une de l'autre : celle de l'eau était assurée par un bain-marie à circulation (puissance de chauffe $1 \mathrm{KW}$ ) thermostaté à $\pm 0,1^{\circ} \mathrm{C}$; celle du fluide encrassant était assurée par le préchauffeur situé en amont immédiat de la maquette et mesurée à l'aide d'un thermomètre à $\pm 0,5^{\circ} \mathrm{C}$ à la sortie de celle-ci.

\subsection{Prototype}

Le prototype était un échangeur industriel (Vicarb, modèle V 7) à plaques de dimensions $150 \times 500 \mathrm{~mm}$ espacées de $3,8 \mathrm{~mm}$. Il comportait un préchauffeur (zone de récupération de chaleur à $93 \%$ ), une zone de chauffage et une section de chambrage. Le produit préchauffé était porté à sa température de traitement (régulation électronique à $\pm 0,3^{\circ} \mathrm{C}$ )dans la zone de chauffage où l'énergie calorifique était fournie par un courant d'eau chaude de débit $1000 \pm 51 \cdot \mathrm{h}^{-1}$.

Ce prototype est alimenté par une pompe volumétrique (P.C.M.) à débit variable $\left(0\right.$ à $\left.12001 . h^{-1}\right)$ et régulé à \pm 1 1.h.' . 


\subsection{Evaluation des caractéristiques thermiques}

Il était essentiel pour l'installation de laboratoire de connaître le profil thermique dans le préchauffeur d'une part, et les variations de température dans la maquette d'autre part.

- Profil thermique

Le flux de chaleur échangé entre l'eau du bain-marie et le fluide circulant dans le tube du préchauffeur est donné par la relation classique suivante :

$$
\varnothing=\frac{A \cdot \alpha \cdot\left[\left(t_{0}-t_{1}\right)-\left(t_{0}-t_{2}\right)\right]}{\log _{e} \frac{t_{0}-t_{1}}{t_{0}-t_{2}}}=\rho Q C_{p}\left(t_{2}-t_{1}\right)
$$

$\varnothing=$ flux de chaleur (W).

$A=$ aire d'échange $\left(\mathrm{m}^{2}\right)=2 \pi . \mathrm{r} . l(\mathrm{r}=$ rayon du tube et $l=1$ longueur du tube en $\mathrm{m}$ ).

$\alpha=$ coefficient global d'échange $\left(\mathrm{W} \cdot \mathrm{m}^{-2} \cdot \mathrm{K}^{-1}\right)$.

$\mathrm{Q}=$ débit volumique du fluide $\left(\mathrm{m}^{3} \cdot \mathrm{s}^{-1}\right)$.

$\rho=$ masse volumique $\left(\mathrm{kg} \cdot \mathrm{m}^{-3}\right)$.

$\mathrm{C}_{\mathrm{p}}=$ chaleur spécifique du fluide $\left(\mathrm{J} \cdot \mathrm{kg}^{-1} \cdot \mathrm{K}^{-1}\right)$.

$\mathrm{t}_{0}=$ température du bain'marie $\left({ }^{\circ} \mathrm{C}\right)$.

$\mathrm{t}_{1}=$ température du fluide à l'entrée $\left({ }^{\circ} \mathrm{C}\right)$.

$\mathrm{t}_{2}=$ température du fluide à la sortie $\left({ }^{\circ} \mathrm{C}\right)$.

Cette relation n'est rigoureusement valable que pour un écoulement en régime turbulent. Or la plupart de nos essais seront réalisés à des débits correspondant à un régime laminaire dans le préchauffeur. Aussi, pour obtenir une bonne approximation dans les calculs, $\alpha$ était déterminé expérimentalement à partir de la relation (1) par mesure de la température $t_{2}$ de l'eau pour $t_{1}$ compris entre 15,4 et $16,3^{\circ} \mathrm{C}$ et $\mathrm{t}_{0}$ variable de 27 à $60^{\circ} \mathrm{C}$. Le débit était de $101 . \mathrm{h}^{-1}$. Le tube, de diamètre intérieur $7 \mathrm{~mm}$, avait une longueur de $456 \mathrm{~mm}$.

La relation (1) permettait alors d'exprimer la température $\mathrm{t} d \mathrm{u}$ fluide en fonction de la position dans le préchauffeur, définie par la distance $l$ mesurée à partir de l'entrée :

$$
\begin{gathered}
\mathrm{t}_{l}=\mathrm{t}_{0}-\left(\mathrm{t}_{0}-\mathrm{t}_{1}\right) \exp (-\mathrm{kl})(1 \mathrm{bis}) \\
\text { avec } \mathrm{k}=\frac{2 \pi \mathrm{r} \alpha}{\rho \mathrm{QC}_{\mathrm{p}}}
\end{gathered}
$$

Les profils de température étaient tracés suivant la relation (1 bis) pour un débit constant $\left(101 . \mathrm{h}^{-1}\right)$ avec $\mathrm{t}_{0}$ variable $\left(55-72-80-95^{\circ} \mathrm{C}\right)$ ainsi que pour $t_{0}$ constant $\left(98^{\circ} \mathrm{C}\right)$ avec un débit variable $(10-20-100$ 200 1.h ${ }^{-1}$ ). 
- Variation de température dans la maquette

L'écart de température entre l'entrée $\left(t_{2}\right)$ et la sortie $\left(t_{3}\right)$ de la maquette était calculé en utilisant la relation (l) dans laquelle nous avions fixé :

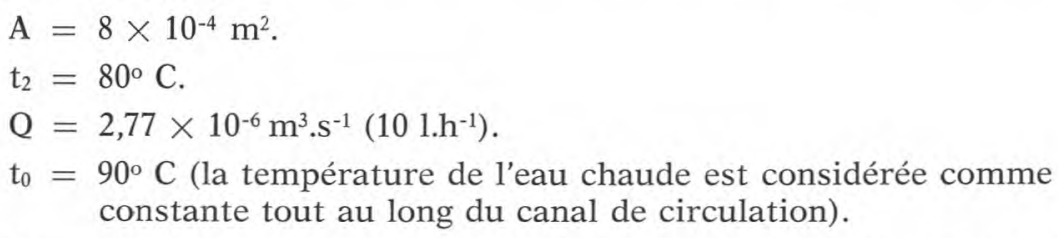

Ces valeurs correspondent à des conditions moyennes pour les essais d'encrassement.

$\alpha$ était calculé à partir des valeurs des coefficients locaux d'échange de part et d'autre de la surface $\left(\alpha_{1}\right.$ et $\left.\alpha_{2}\right)$, de l'épaisseur $\left(e_{p}=0,4 \times 10^{-3}\right.$ $\mathrm{m})$ et de la conductibilité thermique $\left(\lambda_{\mathrm{p}}=15 \mathrm{~W} \cdot \mathrm{m}^{-1} \cdot \mathrm{K}^{-1}\right)$ de l'acier inoxydable par la relation :

$$
\frac{1}{\alpha}=\frac{1}{\alpha_{1}}+\frac{1}{\alpha_{2}}+\frac{\mathrm{e}_{\mathrm{p}}}{\lambda_{\mathrm{p}}}(2)
$$

$\alpha_{1}$ et $\alpha_{2}$ étaient déterminés à partir des relations liant les nombres de Nusselt, Reynolds et Prandtl données par Rohsenow et Hartnett (1973 a).

\subsection{Evaluation des caractéristiques hydrodynamiques}

- Détermination des pertes de charges.

L'écoulement d'un fluide dans une conduite de section donnée et constante peut être décrit par deux nombres sans dimension (ou invariants de similitude) : le nombre de Reynolds (Re) et le nombre de Darcy (Da).

$$
\begin{aligned}
\mathrm{Re} & =\frac{\mathrm{D} \rho \mathrm{V}}{\eta} \\
\mathrm{Da} & =\frac{2 \mathrm{D}}{\rho \mathrm{V}^{2}} \frac{\Delta \mathrm{P}}{\Delta l} \\
\mathrm{D} & =\text { diamètre hydraulique }(\mathrm{m}) \\
\rho & =\text { masse volumique du fluide }\left(\mathrm{kg} \cdot \mathrm{m}^{3}\right) \\
\mathrm{v} & =\text { vitesse moyenne de passage du fluide }\left(\mathrm{m} \cdot \mathrm{s}^{-1}\right) \\
\mathrm{n} & =\text { viscosité dynamique du fluide }(\mathrm{Pa} . \mathrm{s}) \\
\frac{\mathrm{P}}{\Delta l} & =\text { perte de charge par unité de longueur }\left(\mathrm{Pa} \cdot \mathrm{m}^{-1}\right)
\end{aligned}
$$


Ces deux nombres sont liés par une relation :

$\mathrm{Da}=\mathrm{f}(\mathrm{Re})$

qui dépend de la géométrie du canal d'écoulement.

Nous avons tenté de caractériser la maquette et le prototype sur le plan hydrodynamique en déterminant expérimentalement la fonction (5).

Le diamètre hydraulique du prototype et de la maquette était calculé suivant la relation :

$$
\begin{aligned}
\mathrm{D} & =\frac{4 \times \text { section droite }}{\text { périmètre mouillé }}=\frac{2 \mathrm{e} 1}{\mathrm{e}+1} \\
\mathrm{e} & =\text { épaisseur du canal }(\mathrm{m}) . \\
\mathrm{l} & =\text { largeur du canal }(\mathrm{m}) . \\
\mathrm{e}) & \text { et la vitesse moyenne par : } \\
\mathrm{v}= & \frac{\mathrm{Q}}{\text { section }}=\frac{-}{\mathrm{e}}
\end{aligned}
$$

Pour étudier expérimentalement la relation (5), nous mesurions les pertes de charge $(\Delta \mathrm{P})$ en fonction du débit $(\mathrm{Q})$ en utilisant de l'eau à 12 et $19^{\circ} \mathrm{C} . \Delta \mathrm{P}$ était mesuré à l'aide d'un capteur de différence de pression (Schaevitz type 2142-001) placé entre l'entrée et la sortie du prototype ou de la maquette (épaisseur de canal variable de 0,1 à 1,47 mm). L'étendue de mesure du capteur était de 1 bar et sa précision de 0,0025 bar.

Le débit, variable de 0 à $6501 . \mathrm{h}^{-1}$ pour la maquette et à $12001 . \mathrm{h}^{-1}$ pour le prototype, était mesuré par pesée de l'eau en fonction du temps (précision 0,1\%).

- Relations entre caractéristiques de fonctionnement :

$$
\text { Re, v, Q, e }
$$

Dans ce paragraphe on considèrera la circulation d'un fluide donné ( $\rho$ et $n$ constants) dans un canal de largeur donnée ( 1 constant).

La transformation de la relation (3) en explicitant D par (6) donne :

$$
\log _{10} \mathrm{Re}=\log _{10} \mathrm{~V}+\log _{10} \frac{2 \rho 1 \mathrm{e}}{\mathrm{n}(1+\mathrm{e})}
$$

Pour e constant, le graphe de la relation (8) en coordonnées logarithmiques est une droite : droite d'isoépaisseur.

La transformation de la relation (8) en remplaçant e par son expression tirée de $\mathrm{Q}=$ e $1 \mathrm{v}$ donne :

$$
\log _{10} \mathrm{Re}=\log _{10} \mathrm{~V}+\log _{10} \frac{2 \mathrm{Ql} \rho}{\eta}-\log _{10}\left(\mathrm{Q}+\mathrm{l}^{2} \mathrm{v}\right)
$$


Pour Q constant, le graphe de la relation (9) en coordonnées logarithmiques est une courbe d'isodébit.

Ces graphes permettront de visualiser les grandeurs caractéristiques du fonctionnement hydrodynamique.

\subsection{Caractéristiques physiques des fluides}

Les caractéristiques physiques nécessaires dans ce travail étaient la masse volumique $(\rho)$, la viscosité dynamique $(n)$ et la chaleur spécificique $\left(C_{p}\right)$. Les lois de variations de ces grandeurs en fonction de la température sont données dans le tableau 1. Elles sont soit tirées directement des travaux de Bertsch (1981) pour le lait, soit calculées à partir d'un ajustement des données de Kessler (1981) pour l'eau et celles de Culioli et al. (1974) et Cabarat (1980) pour le rétentat d'ultrafiltration (x2). En l'absence de données connues pour le lactosérum, ce dernier était assimilé à de l'eau. Ces lois étaient utilisées pour calculer les valeurs des caractéristiques physiques des produits à la température de l'essai si celle-ci était constante. Dans le cas d'une température variable (cas des profils thermiques dans le préchauffeur, par exemple), nous avons utilisé la moyenne géométrique de ces caractéristiques physiques entre les valeurs extrêmes des températures considérées.

\subsection{Essais d'encrassement}

Les fluides encrassants étaient du lactosérum doux de fromagerie de pâte molle et du lait écrémé. Afin de disposer d'un fluide identique à chaque essai, nous le reconstituions à partir de poudre préparée par atomisation (poudre dite «Low Heat » dans le cas du lait). Les compositions chimiques des 2 fluides, calculées à partir des analyses faites sur les poudres, sont données dans le tableau 2. La teneur en substance sèche totale (E.S.T.) était déterminée par dessication à l'étuve à $103 \pm 2^{\circ} \mathrm{C}$ pendant $7 \mathrm{~h}$ (Amariglio et al., 1973). La teneur en matières azotées totales (M.A.T.) était déterminée en dosant l'azote par microKjeldahl et en multipliant le résultat par le coefficient 6,38 (Ogg, 1960). La teneur en cendres était déterminée par incinération de la substance sèche à $530^{\circ} \mathrm{C}$ (Amariglio et al., 1973). Les teneurs en azote non caséinique (N.C.N.) et en azote non protéique (N.P.N.) étaient dosées par la méthode de Aschaffenburg et Drewry (1959). La teneur en protéines solubles totales était calculée par différence entre N.C.N. et N.P.N. La teneur en protéines non dénaturées était calculée comme étant la somme des teneurs en $\beta$-lactoglobuline, a-lactalbumine et Bovine Serum Albumine (B.S.A.), dosées par chromatographie liquide haute pression (H.P.L.C.) en tampon tris-(hydroxyméthyl) aminoéthane $0,01 \mathrm{M}$ à pH 6,68 sur colonne Varian micropak TSK à gel de silice $3000 \mathrm{SW}$. Pour les deux premières protéines, le dosage était effectué sur le lactosérum reconstitué à 63,2 g.kg-1 d'extrait sec total. Pour la dernière (B.S.A.) le lactosérum avait un 
TABLEAU 1

Caractéristiques physiques des fluides

\begin{tabular}{|c|c|c|c|c|}
\hline & $\begin{array}{l}\text { Caractéritique } \\
\text { physique }\end{array}$ & $\begin{array}{l}\text { Domaine de } \\
\text { validité }\end{array}$ & Loi & Référence \\
\hline Eau & $\begin{array}{l}n \\
\mathrm{Cp}\end{array}$ & $\begin{aligned} t & <40^{\circ} \mathrm{C} \\
t & \geqslant 40^{\circ} \mathrm{C} \\
t & \leqslant 20^{\circ} \mathrm{C} \\
20 & <\mathrm{t}<60^{\circ} \mathrm{C} \\
\mathrm{t} & \geqslant 60^{\circ} \mathrm{C} \\
\mathrm{t} & \leqslant 20^{\circ} \mathrm{C} \\
20 & <\mathrm{t}<60^{\circ} \mathrm{C} \\
\mathrm{t} & \geqslant 60^{\circ} \mathrm{C} \\
t & <40^{\circ} \mathrm{C} \\
40 & \leqslant t<60^{\circ} \mathrm{C} \\
\mathrm{t} & \geqslant 60^{\circ} \mathrm{C}\end{aligned}$ & $\begin{array}{l}\rho=1000-0,15 \cdot \mathrm{t} \\
\rho=995-0,5 \cdot(\mathrm{t}-40) \\
\eta=(1,75-0,04 \cdot \mathrm{t}) \cdot 10 \cdot 3 \\
\eta=(1,002-0,01 \cdot(\mathrm{t}-20)) \cdot 10^{-3} \\
\eta=(0,466-0,005 \cdot(\mathrm{t}-60)) \cdot 10^{-3} \\
\mathrm{Cp}=4218-1,5 \cdot \mathrm{t} \\
\mathrm{Cp}=4180 \\
\mathrm{Cp}=4180+0,8 \cdot(\mathrm{t}-60) \\
\lambda=0,569+0,0015 \cdot \mathrm{t} \\
\lambda=0,632+0,0011 \cdot(\mathrm{t}-40) \\
\lambda=0,654+0,0007 \cdot(\mathrm{t}-60)\end{array}$ & $\begin{array}{l}\text { Ajustements } \\
\text { d'après } \\
\text { KESSLER (1981) }\end{array}$ \\
\hline Lait & $\begin{array}{l}n \\
\mathrm{Cp} \\
\lambda\end{array}$ & & 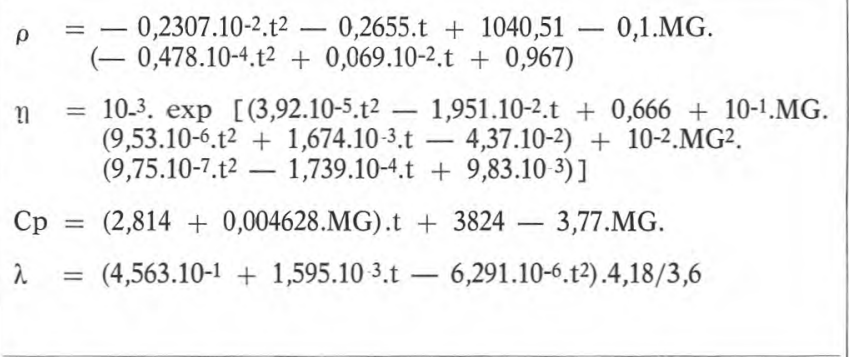 & $\begin{array}{l}\text { BERTSCH (1981) } \\
\text { Ajustement d'après } \\
\text { FERNANDEZ-MARTIN } \\
\text { (1972) }\end{array}$ \\
\hline Rétentat (x2) & $\begin{array}{l}n \\
\mathrm{Cp} \\
\lambda\end{array}$ & $\begin{aligned} t & <10^{\circ} \mathrm{C} \\
10 & \leqslant \mathrm{t}<50^{\circ} \mathrm{C} \\
\mathrm{t} & \geqslant 50^{\circ} \mathrm{C}\end{aligned}$ & $\begin{array}{l}\rho \quad=1050-0,5 \cdot t \\
\eta \quad=6 \cdot 10^{-3} \\
\eta \quad=2,2 \cdot 10^{-3} \\
\eta \quad=10^{-3} \\
\mathrm{Cp}=3525+2,75 \cdot \mathrm{t} \\
\lambda \quad=0,48+10^{-3} \cdot \mathrm{t}\end{array}$ & $\begin{array}{l}\text { Ajustement d'après } \\
\text { CABARAT (1980) } \\
\text { CULIOLI (1974) } \\
\text { CABARAT (1980) } \\
\begin{array}{c}\text { FERNANDEZ-MARTIN } \\
\text { (1972) }\end{array} \\
\text { FERNANDEZ-MARTIN } \\
\text { et MONTES (1972) }\end{array}$ \\
\hline $\begin{array}{l}\rho: \text { masse } v \\
\eta{ }_{\mathrm{Cp}}: \text { viscosité } \\
\text { chaleur }\end{array}$ & $\begin{array}{l}\text { olumique }(\mathrm{kg} . \mathrm{m}- \\
\text { dynamique }(\mathrm{Pa} \text {. } \\
\text { spécifique }\left(\mathrm{J} . \mathrm{kg}^{-}\right.\end{array}$ & & $\begin{array}{l}\lambda \text { : conductivité thermique }\left(\mathrm{W} \cdot \mathrm{m}^{-1} \cdot \mathrm{K}^{-1}\right) \\
\mathrm{t} \text { : température }\left({ }^{\circ} \mathrm{C}\right) \\
\mathrm{MG}: \text { taux de matière grasse }\left(\mathrm{g} \cdot \mathrm{kg}^{-1}\right)\end{array}$ & \\
\hline
\end{tabular}


TABLEAU 2

Compositon (g.kg-1) des fluides d'encrassement reconstitués à partir de poudre

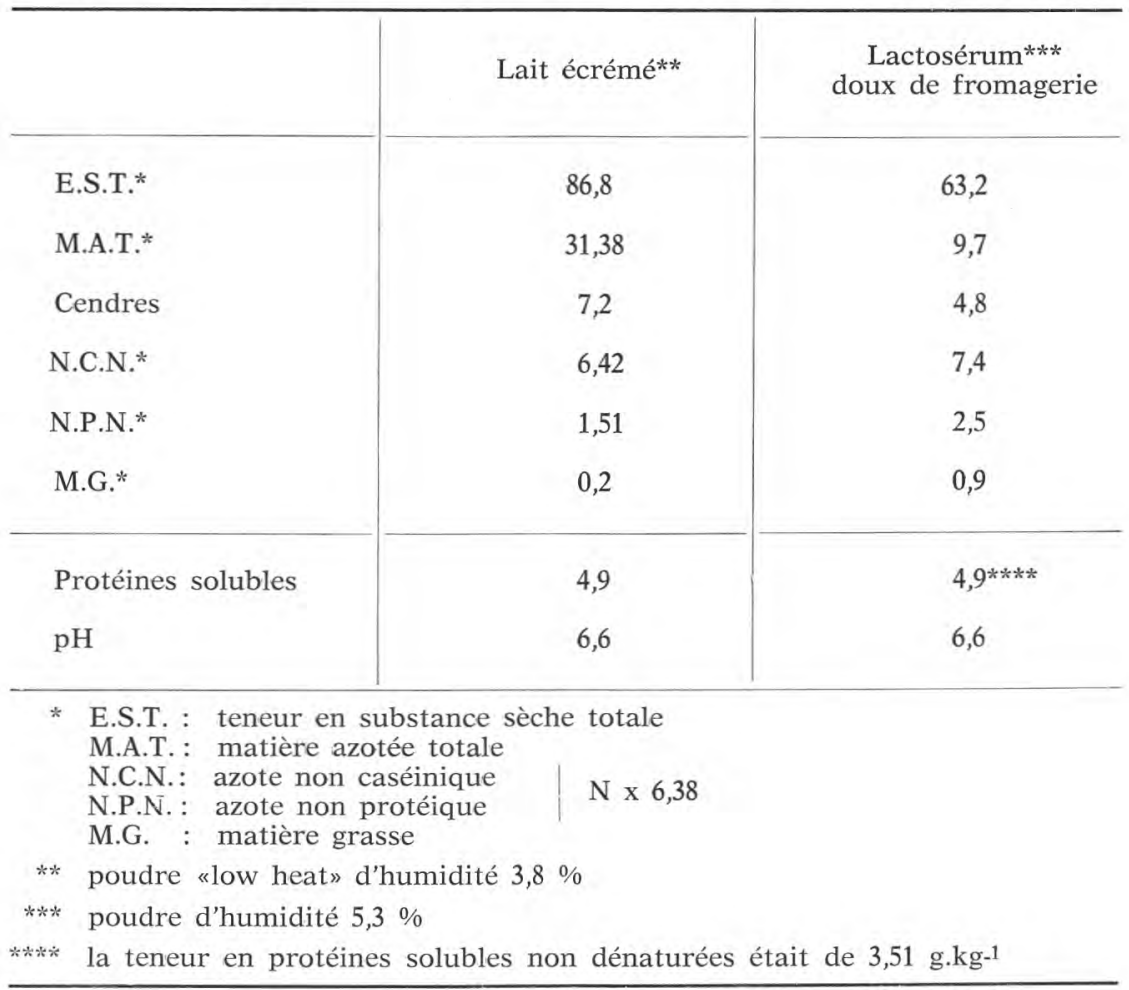

E.S.T. de 200 g. $\mathrm{kg}^{-1}$. La lecture se faisait à $280 \mathrm{~nm}$ de longueur d'onde. La teneur en matière grasse (M.G.) était déterminée à l'aide de la méthode acido-butyrométrique (décrite pour le lactosérum sec par Amariglio et al., 1973) modifiée : le produit était reconstitué à 4 fois son extrait sec normal et on utilisait un butyromètre à lait écrémé.

La surface d'étude était en acier inoxydable du type $18 \% \mathrm{Cr}$ $10 \% \mathrm{Ni}$, polie mécaniquement au papier de granulométrie $12 \mu \mathrm{m}$.

Chaque essai était réalisé en circuit ouvert, c'est-à-dire sans recyclage du fluide, que ce soit avec l'installation de laboratoire ou avec le prototype. La première phase de l'essai consistait à équilibrer l'installation ou le prototype, aux plans hydrodynamique et thermique, en utilisant de l'eau. Ensuite, l'essai d'encrassement était effectué en faisant circuler le lait ou le lactosérum. Différents essais réalisés avec la maquette, ont permis de faire varier les paramètres suivants : durée (2 à $90 \mathrm{~min})$; température de traitement (60 à $85^{\circ} \mathrm{C}$ ) ; écart de température avec l'eau circulant le long de l'autre face de 
l'échantillon $\left(-15\right.$ à $\left.+15^{\circ} \mathrm{C}\right)$. Au cours de ces essais, le débit de lait était $51 . \mathrm{h}^{-1}$ pour une épaisseur de canal de $4 \times 10^{-3} \mathrm{~m}$. Pour le lactosérum, le débit était de $101 . \mathrm{h}^{-1}$ pour une épaisseur de canal de $1,47 \times 10^{-3} \mathrm{~m}$. Dans le prototype, le débit était de $3001 \cdot \mathrm{h}^{-1}$. La température de traitement était 72 ou $95^{\circ} \mathrm{C}$. Dans la zone de récupération de chaleur, l'écart de température entre le produit traité et le produit se réchauffant était quasi-constant et voisin de $5^{\circ} \mathrm{C}$. Dans la zone de chauffage, l'écart de température entre l'eau chaude et le produit variait de $9^{\circ} \mathrm{C}$ à l'entrée à $1^{\circ} \mathrm{C}$ à la sortie. Dans la zone de chambrage l'écart de température était quasi-nul (Desmares, 1982). Après un rinçage à l'eau, l'échangeur (maquette ou prototype) était démonté.

Le dépôt formé lors de l'essai d'encrassement était caractérisé par sa masse (pesée à $\pm 2 \times 10^{-5}$ et à $\pm 5 \times 10^{-3} \mathrm{~g}$ respectivement pour la maquette et le prototype) après mise en équilibre avec l'atmosphère ambiante pendant $24 \mathrm{~h}$. En outre, il faisait l'objet d'une observation au microscope de métallographie.

\section{RESULTATS}

\subsection{Caractéristiques thermiques}

Les mesures de température effectuées au cours des essais de transferts de chaleur dans le préchauffeur permettent de calculer (relation (1)) une valeur moyenne du coefficient global d'échange égale à $1004 \mathrm{~W} \cdot \mathrm{m}^{-2} \cdot \mathrm{K}^{-1}$. Utilisant cette valeur dans la relation (1 bis) nous calculons les profils de température pour du lait écrémé circulant dans le préchauffeur. La figure 2 représente les profils pour un débit de $101 . h^{-1}$ à différentes températures de bain-marie de préchauffage. La température augmente rapidement dans le première mètre du tube puis plus lentement; à partir de $4 \mathrm{~m}$, l'écart entre la température du lait et celle du bain-marie est négligeable (inférieure à $0,03^{\circ} \mathrm{C}$, résultat logique puisqu'il correspond à une efficacité d'échange $\frac{t_{2}-t_{1}}{t_{0}-t_{1}}$ égale à 0,999). Par contre, lorsque les débits sont élevés, les écarts peuvent atteindre plusieurs dizaines de degrés à cette même distance de $4 \mathrm{~m}$ (figure 3).

Au cours des essais, le préchauffeur subit lui-même un encrassement qui abaisse ses performances et nécessite de réguler la température du bain-marie de préchauffage. (Un essai de $3 \mathrm{~h}$ avec du lactosérum circulant à un débit de $101 . \mathrm{h}^{-1}$, nécessite une augmentation de $3^{\circ} \mathrm{C}$ de la température du bain-marie de préchauffage entre le début et la fin de l'essai pour maintenir la température de sortie du produit à $80^{\circ} \mathrm{C}$ ). Ceci entraîne la nécessité de nettoyer le préchauffeur entre chaque essai, même de courte durée, pour assurer au produit traité un profil thermique quasi-constant et reproductible. 

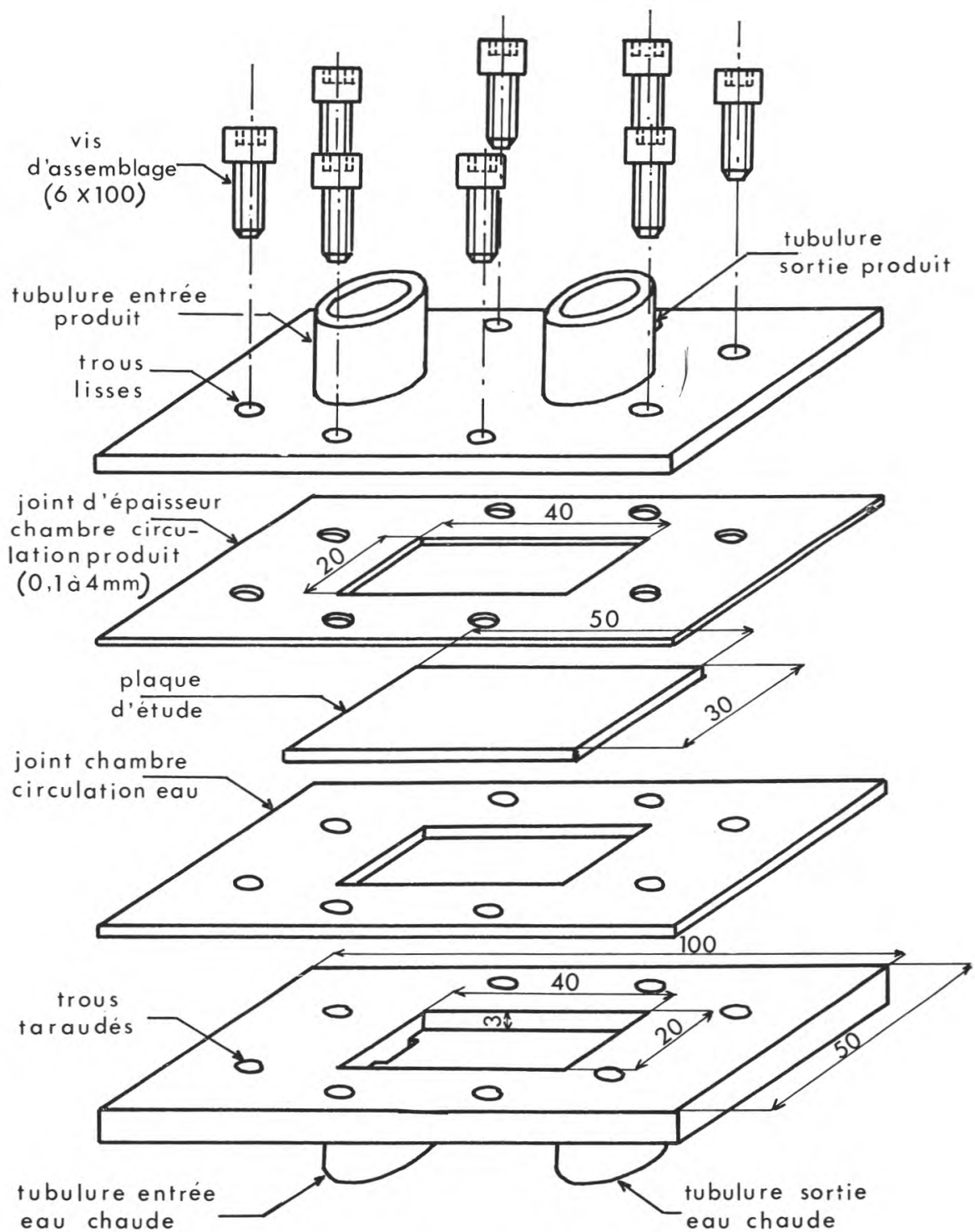

fig. 1a

Schéma éclaté de l'échangeur expérimental (cotes fonctionnelles en $\mathrm{mm}$ ).

Schema of the experimental heat exchanger (fonctionnal sizes in $\mathrm{mm}$ ). 


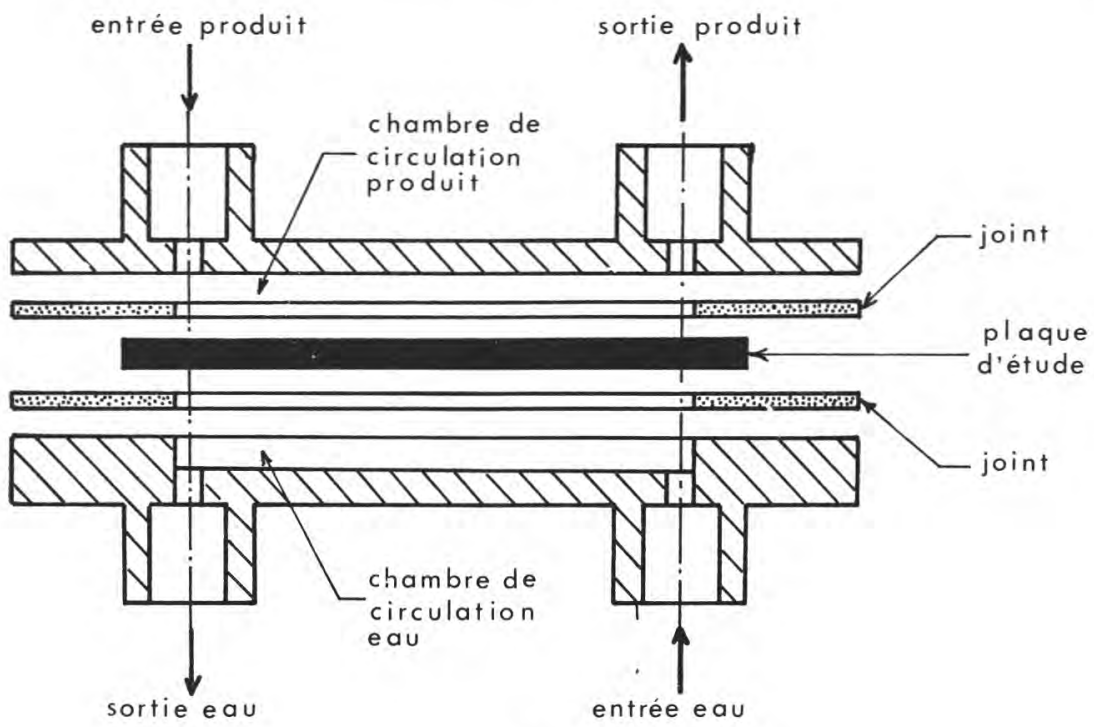

fig. 1b

Coupe longitudinale de l'échangeur expérimental.

Longitudinal cut through the experimental heat exchanger.

Dans la maquette, dans les conditions indiquées précédemment (2.3.), la valeur calculée de $\alpha$ est de $2400 \mathrm{~W} \cdot \mathrm{m}^{-2} \cdot \mathrm{K}^{-1}$ et la variation de température du produit entre l'entrée et la sortie, voisine de $1^{\circ} \mathrm{C}$.

\subsection{Caractéristiques hydrodynamiques}

Les variations de $\log _{\mathrm{e}} \mathrm{Da}$ en fonction de $\log _{\mathrm{e}} \mathrm{Re}$ sont représentées sur la figure 4 pour le prototype et pour la maquette à différentes épaisseurs de canal. Pour la maquette, les mesures de pression n'ont pu être réalisées exactement à l'entrée et à la sortie de la chambre de traitement thermique en raison des difficultés matérielles d'implantation des organes de mesure. Nous avons donc calculé, sur la base des données de Idel'cik (1969), la contribution des pertes de charge dans les tubulures et des pertes de charge singulières dans les coudes, rétrécissements et élargissements (fig. 1a et $1 \mathrm{~b}$ ) situés entre les points de mesure et la chambre d'étude. Pour des épaisseurs de canaux allant de 0,1 à $0,4 \mathrm{~mm}$, ces pertes de charge ne dépassent pas $5 \%$ de la perte de charge mesurée. Par contre, pour le canal d'épaisseur 1,47 $\mathrm{mm}$, ces pertes de charges deviennent prépondérantes. En conséquence, dans ce cas, les valeurs mesurées ne seront pas interprétables. 


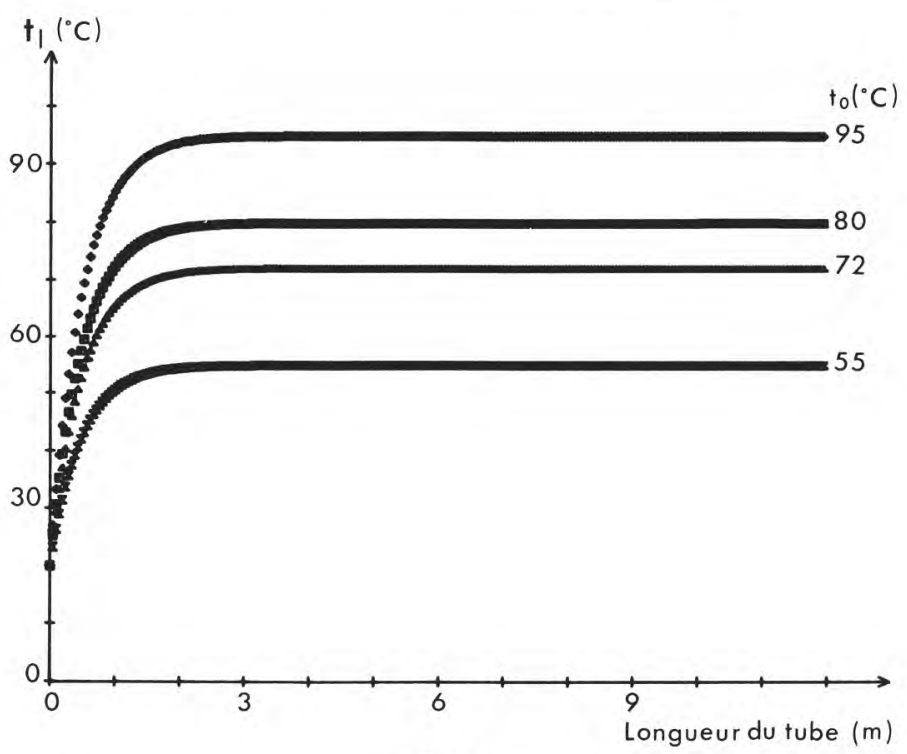

fig. 2

Profils de température (t $t)$, calculés à partir de la relation ( 1 bis) pour du lait écrémé circulant à $101 \mathrm{~h}^{-1}$ dans le préchauffeur tubulaire (diamètre $=7,0 \mathrm{~mm}$; coefficient global d'échange $\alpha=1004 \mathrm{~W} \cdot \mathrm{m}^{-2} \cdot \mathrm{K}^{-1}$ ) à différentes températures de bain-marie $\left(\mathrm{t}_{\mathrm{o}}\right)$.

Temperature profiles ( $\mathrm{t} l$ ) calculated from relation (1 bis) for skim-milk flowing at $101 . h^{-1}$ in the tubular pre-heater (diameter $=7,0 \mathrm{~mm}$; global heat transfer coefficient $\left.\alpha=1004 \mathrm{~W} \cdot \mathrm{m}^{-2} \cdot \mathrm{K}^{-1}\right)$ for some double saucepan temperature $\left(t_{0}\right)$.

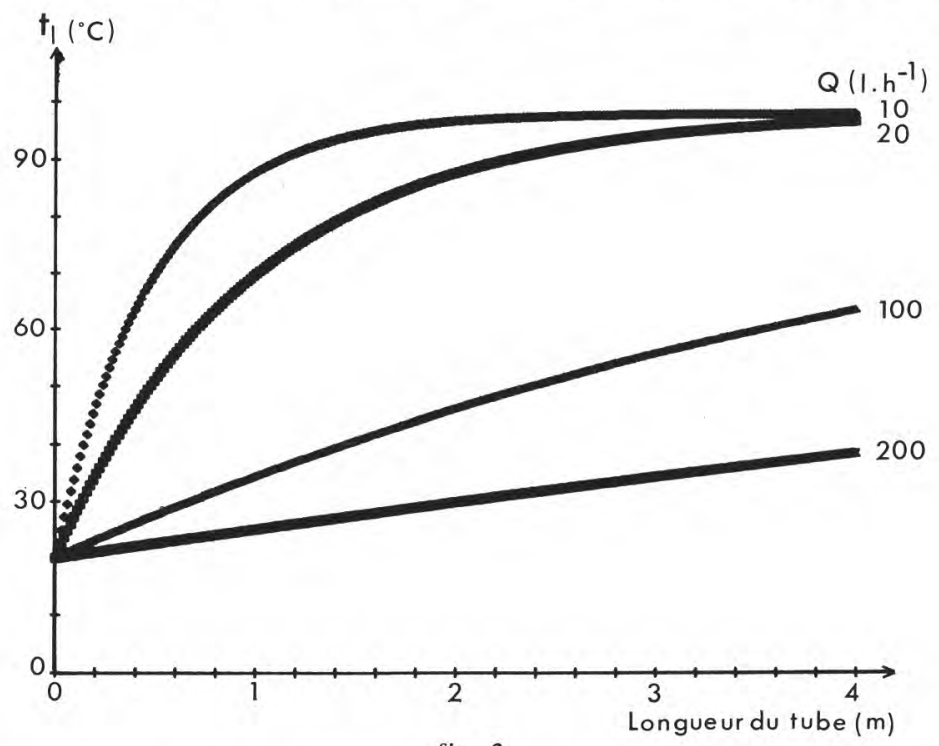

fig. 3

Profils de température $(\mathrm{t} l)$, calculés à partir de la relation $(1 \mathrm{bis})$ pour du lait écrémé circulant dans le préchauffeur tubulaire (diamètre $=7,0 \mathrm{~mm} ; \alpha=1004$ W.m-2. $\mathrm{K}^{-1}$ ) à différents débits $(\mathrm{Q})$ pour une température de bain-marie de $98^{\circ} \mathrm{C}$. Temperature profiles ( $\mathrm{t} l$ ) calculated from relation $(1$ bis) for skim-milk flowing in the tubular pre-heater (diameter $=7,0 \mathrm{~mm}$; global heat transfer coefficient $\left.\alpha=1004 \mathrm{~W} \cdot \mathrm{m}^{-2} \cdot \mathrm{K}^{-1}\right)$ at some flows $(Q)$ for double saucepan temperature of $98^{\circ} \mathrm{C}$. 


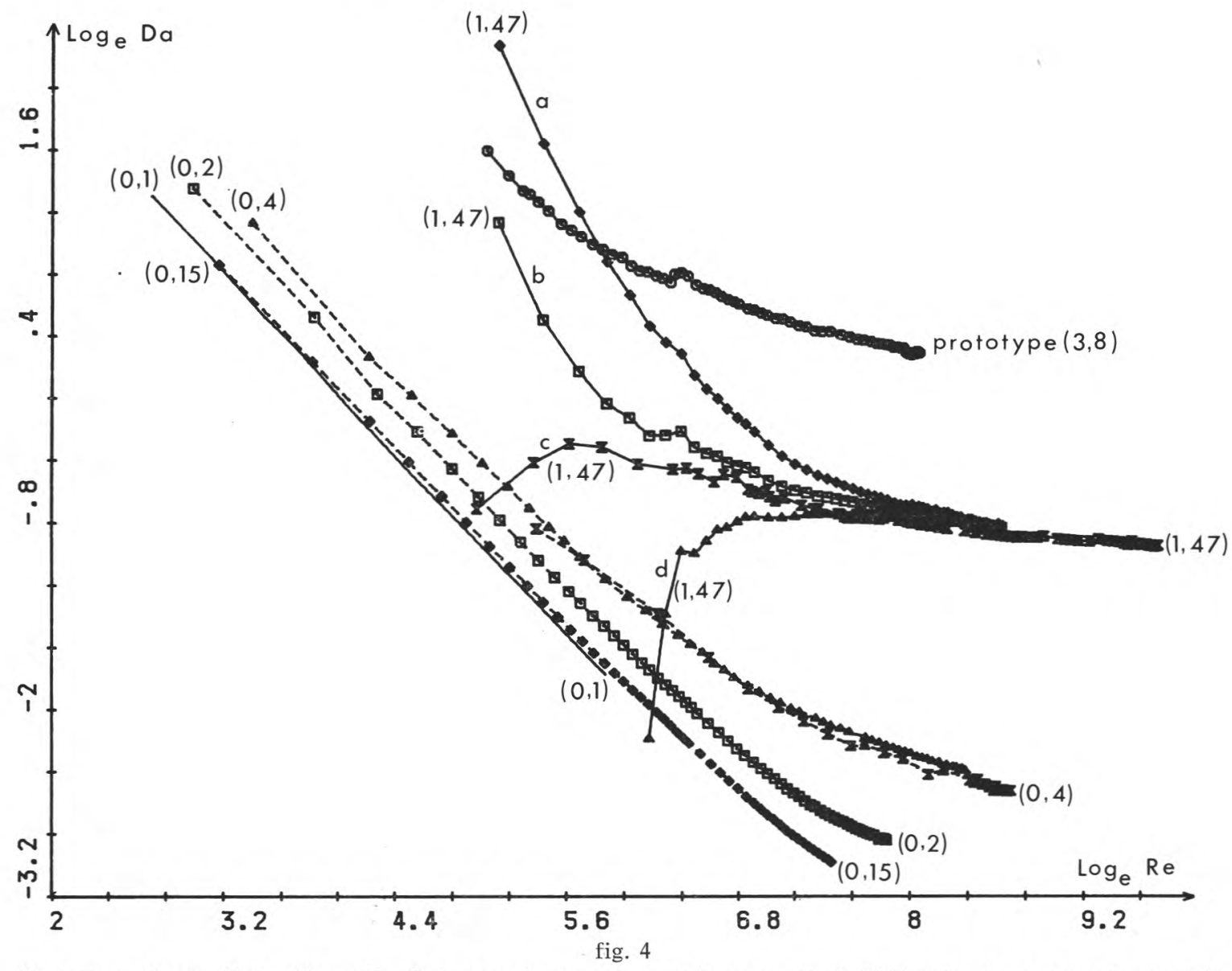

Variations Darcy (Da) - Reynolds (Re) pour le prototype et la maquette à différentes épaisseurs de canal ( en $\mathrm{mm}$ (largeur $=20 \mathrm{~mm}$ ). (Courbes $\mathrm{a}, \mathrm{b}, \mathrm{c}, \mathrm{d}$ : répétition du même essai avec 4 valeurs différentes du zéro du capteur de différence de pression).

$\log _{e}$ Darcy ( $\mathrm{Da}$ ) versus $\log _{e}$ Reynolds (Re) for the prototype and the model with some channel thickness ( in $\mathrm{mm}$ (width $=20 \mathrm{~mm}$ ). (Curves $a, b, c, d$ : repetition of the same experience with 4 different electrical zero values for pressure difference sensor). 


\section{TABLEAU 3}

Valeurs des coefficients $\mathrm{k}$ et $\mathrm{m}$ de la relation entre le nombre de Darcy (Da) et le nombre de Reynolds (Re) : $\mathrm{Da}=\mathrm{k} \cdot \mathrm{Re}^{\mathrm{m}}$ et valeurs de $\mathrm{Re}$ pour les changements de régime, pour le prototype et la maquette à différentes épaisseurs de canal

\begin{tabular}{|c|c|c|c|c|c|c|c|}
\hline \multirow{3}{*}{ Type d'échangeur } & \multirow{3}{*}{$\begin{array}{l}\text { Epaisseur du canal } \\
(\mathrm{mm})\end{array}$} & \multicolumn{6}{|c|}{ Régime et changement de régime } \\
\hline & & \multicolumn{2}{|c|}{ Laminaire } & \multirow{2}{*}{$\begin{array}{c}\text { Fin de laminaire } \\
\text { Re critique }\end{array}$} & \multicolumn{2}{|c|}{ Turbulent } & \multirow{2}{*}{$\frac{\text { Début de turbulent }}{\operatorname{Re}}$} \\
\hline & & $\mathrm{k}$ & $\mathrm{m}$ & & $\mathrm{k}$ & $\mathrm{m}$ & \\
\hline $\begin{array}{l}\text { Prototype Vicarb } \\
\text { type V7 ; } 7 \text { canaux }\end{array}$ & 3,8 & - & - & - & 7 & $-0,2$ & 970 \\
\hline \multirow[t]{4}{*}{ Maquette } & 0,40 & 74 & $-0,93$ & 270 & - & - & - \\
\hline & 0,20 & 74 & $-0,99$ & 303 & - & - & - \\
\hline & 0,15 & 47,5 & $-0,95$ & 650 & - & - & - \\
\hline & 0,10 & 50 & $-0,97$ & $>400$ & - & - & - \\
\hline
\end{tabular}


Pour la maquette, les courbes $\log _{e} \mathrm{Da}$ en fonction de $\log _{e}$ Re présentent toutes, dans leur première partie, une partie linéaire, traduisant une relation du type :

$$
\mathrm{Da}=\mathrm{kRe}^{\mathrm{m}}
$$

L'analyse par régression linéaire de ces portions de courbes a permis de calculer les valeurs des coefficients $\mathrm{k}$ et $\mathrm{m}$ (tableau 3) ainsi qu'une valeur critique de $\mathrm{Re}$ pour laquelle la relation (10) cesse d'être vérifiée.

Les valeurs de $\mathrm{m}$ sont très voisines de -1 , celles de $\mathrm{k}$ varient de 50 à 74 .

Les valeurs critiques de $R e$ varient de $650(\mathrm{e}=0,15 \mathrm{~mm})$ à 270 (e $=0,4 \mathrm{~mm})$.

Dans le prototype, pour des Re supérieurs à $970\left(\log _{\mathrm{e}} \mathrm{Re}=6,88\right)$, la courbe (figure 4) présente une partie linéaire $(\mathrm{k}=7, \mathrm{~m}=-0,2)$.

Les relations (8) et (9) entre nombre de Reynolds (Re), vitesse moyenne de passage (v), épaisseur de canal (e) et débit (Q) appliquées à de l'eau à $19^{\circ} \mathrm{C}$ circulant dans la maquette sont représentées graphiquement sur la figure 5 .

Les droites d'isoépaisseur ont été tracées pour des valeurs discrètes de e entre 0,1 et $4 \mathrm{~mm}$ et les courbes d'isodébit couvrent un domaine de 1 à 300 l.h. . $^{-1}$.

Le report des valeurs de Re critiques indiquées dans le tableau 3 permet de situer sur la figure 5 certaines limites particulières de changement de régimes d'écoulement.

\subsection{Encrassement}

La dispersion des résultats a été étudiée à l'aide d'essais d'encrassement par du lactosérum, répétés 5 fois dans les conditions hydrodynamiques et thermiques suivantes : $\mathrm{Q}=10 \mathrm{1. \textrm {h } ^ { - 1 }}$; préchauffeur de $2 \mathrm{~m} ; \mathrm{e}=1,5 \mathrm{~mm} ; \mathrm{v}=9,4 \times 10^{-2} \mathrm{~m} \cdot \mathrm{s}^{-1} ; \mathrm{t}=80^{\circ} \mathrm{C} ; \Delta \mathrm{t}=+10^{\circ} \mathrm{C}$. Nous avons constaté que la dispersion était d'autant plus forte que l'encrassement est important. Ainsi, dans un essai d'une durée de $5 \mathrm{~min}$, la masse moyenne de dépôt $(\mathrm{m})$ est $63 \mu \mathrm{g} . \mathrm{cm}^{-2}$ et l'écart type $(\sigma) 9 \mu \mathrm{g} . \mathrm{cm}^{-2}$. Pour $30 \mathrm{~min}, \mathrm{~m}$ et $\sigma$ deviennent respectivement 950 et $40 \mu \mathrm{g} . \mathrm{cm}^{-2}$.

Les résultats des essais d'encrassement réalisés avec le préchauffeur de $4 \mathrm{~m}$, dans des conditions connues $\left(\mathrm{v}=9,4 \times 10^{-2} \mathrm{~m} . \mathrm{s}^{-1}\right.$, Re voisin de 700 pour le lactosérum ; v $=1,73 \times 10^{-2} \mathrm{~m} . \mathrm{s}^{-1}$ et $\mathrm{Re}$ voisin de 190 pour le lait) sont reportés sur les figures 6, 7 et 8 . Pour les deux produits à $80^{\circ} \mathrm{C}$ et pour un écart de température $\Delta \mathrm{t}=+10^{\circ} \mathrm{C}$, le dépôt se forme au bout de durées relativement courtes (figure 6) ; ainsi pour le lactosérum, 80 ug de dépôt se sont déjà formés sur $1 \mathrm{~cm}^{2}$ de surface après 5 min d'essai. Pour ce produit, la vitesse d'encrassement est lente pendant environ $50 \mathrm{~min}$ et augmente ensuite très sensiblement. Pour le lait, la masse de dépôt augmente beaucoup plus rapidement avec la durée, dès le début du traitement. Dans les 


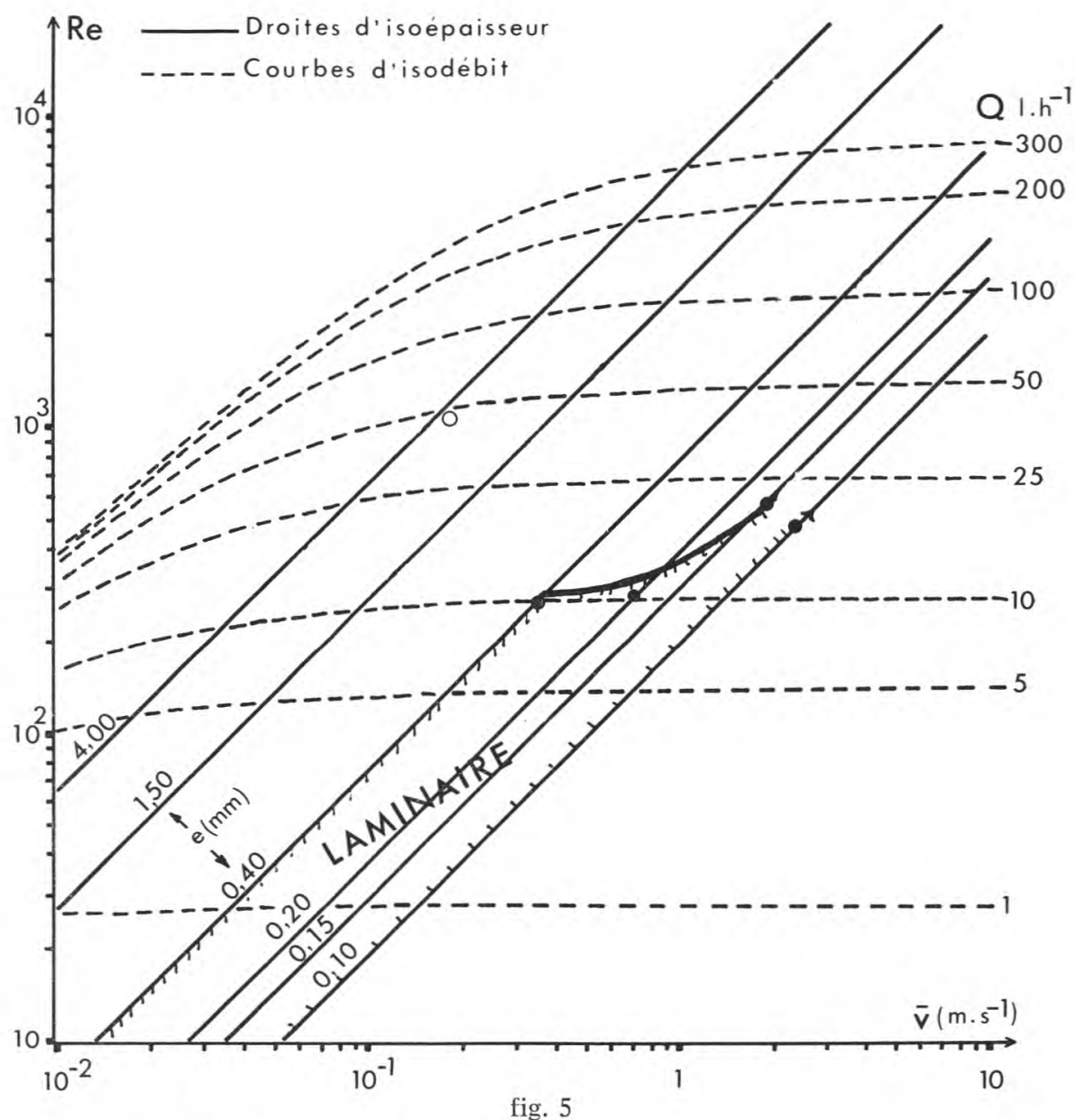

Relations entre nombre de Reynolds (Re), vitesse moyenne de passage ( $v$ en $\left.\mathrm{m} . \mathrm{s}^{-1}\right)$, épaisseur de canal (e en $\mathrm{mm}$ ) et débit $\left(\mathrm{Q}\right.$ en $\left.1 . \mathrm{h}^{-1}\right)$ pour de l'eau à $19^{\circ} \mathrm{C}$ circulant dans la maquette de largeur de canal $1_{\mathrm{m}}=20 \mathrm{~mm}$; domaines de régime hydrodynamique : - Re critique de fin de régime laminaire pour la maquette ; O Re de début de régime turbulent pour le prototype.

Relations between Reynolds number (Re), mean flow rate ( $v$ in $\left.m \cdot s^{-1}\right)$, channel thickness (e in $\mathrm{mm}$ ) and flow (Q in $\left.1 . \mathrm{h}^{-1}\right)$ for water at $19^{\circ} \mathrm{C}$ flowing through the model with width channel $l_{m}=20 \mathrm{~mm}$.; hydrodynamical flow areas: $\bullet$ Critical Re for the end of laminar flow in the model; 0 Re for the beginning of turbulent flow in the prototype.

mêmes conditions d'écoulement, l'augmentation de la température de traitement du lait (fig. 7) et celle du $\Delta t$ (fig. 8) accroissent fortement la quantité de dépôt. 


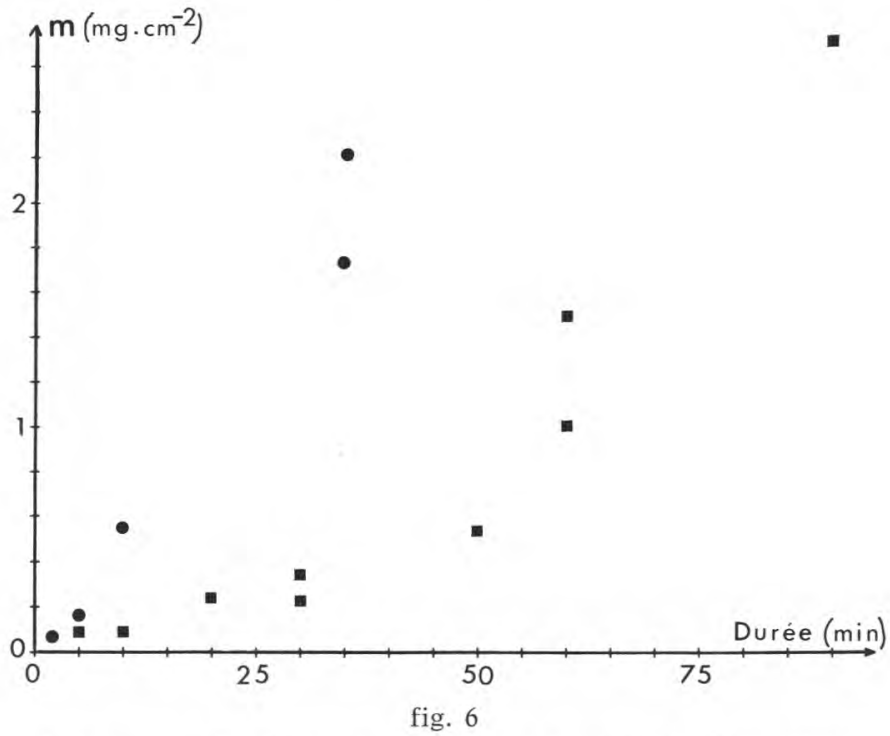

Evolution de la masse de dépôts $(\mathrm{m})$ sur acier inoxydable $18 \mathrm{Cr}-10 \mathrm{Ni}$ en fonction de la durée de contact; $\mathrm{t}=80^{\circ} \mathrm{C}$ et $\Delta \mathrm{t}=10^{\circ} \mathrm{C}$; préchauffeur de $4 \mathrm{~m}$.

(•) Lait écrémé $\left(\mathrm{Q}=51 . \mathrm{h}^{-1} ; \mathrm{v}=1,73 \times 10^{-2} \mathrm{~m} \cdot \mathrm{s}^{-1} ; \mathrm{Re} \simeq 190\right)$.

(匹) Lactosérum $\left(\mathrm{Q}=101 . \mathrm{h}^{-1} ; \mathrm{v}=9,4 \times 10^{-2} \mathrm{~m} \cdot \mathrm{s}^{-1}\right.$; $\left.\mathrm{Re} \simeq 700\right)$.

Fouling mass $(\mathrm{m})$ on $304 \mathrm{~L}$ stainless steel versus contact time; $t=80^{\circ} \mathrm{C}$ and $\Delta t=10^{\circ} \mathrm{C}$; preheater with $4 \mathrm{~m}$ length.

(•) Skim-milk $\left(Q=51 . \mathrm{h}^{-1} ; v=1,73 \times 10^{-2} \mathrm{~m} \cdot \mathrm{s}^{-1} ; \quad R e \simeq 190\right)$.

(1) Sweet whey $\left(Q=101 . \mathrm{h}^{-1} ; v=9,4 \times 10^{-2} \mathrm{~m}^{-1} \mathrm{~s}^{-1} ; \mathrm{Re} \simeq 700\right)$.

L'observation au microscope révèle l'existence d'un film mince (épaisseur inférieure à $0,5 \mu \mathrm{m}$, non mesurable sur notre microscope) formé au cours des premiers instants de contact. Ensuite, lorsque les durées de contact sont plus longues, un dépôt plus épais apparaît. Il est pulvérulent et peu adhérent pour le lactosérum et floconneux et fortement adhérent pour le lait. Le dépôt présente en outre une structure alvéolaire lorsque les essais sont réalisés à température élevée et lorsque la vitesse d'écoulement est faible : cas des essais sur lait ; cas des zones mortes du canal d'écoulement en particulier le long du joint. Ces observations globales sont également valables pour les dépôts formés après quelques heures de traitement thermique sur le prototype travaillant en régime turbulent $\left(\mathrm{v}=0,14 \mathrm{~m} \cdot \mathrm{s}^{-1}\right.$ et $\operatorname{Re}$ voisin de 1000 ), sur des plaques dans la zone de température 70 à $95^{\circ} \mathrm{C}$.

Lorsque les conditions d'écoulement du lait changent dans la maquette $\left(\operatorname{Re}=740, \mathrm{v}=0,7 \mathrm{~m} \cdot \mathrm{s}^{-1}\right)$ à la suite d'une réduction de 


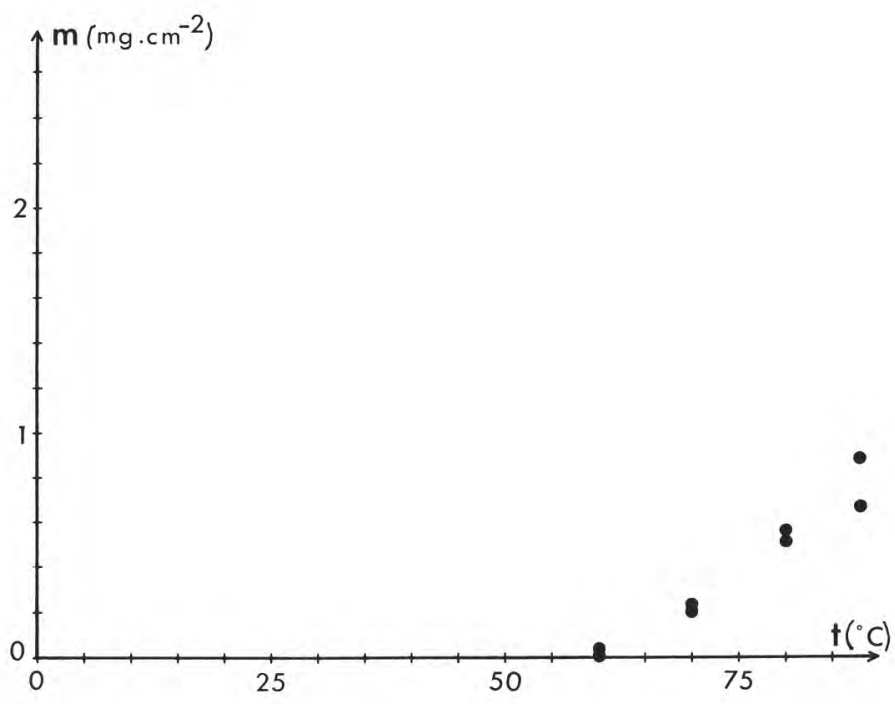

fig. 7

Evolution de la masse de dépôt $(\mathrm{m})$ sur acier inoxydable $18 \mathrm{Cr}-10 \mathrm{Ni}$ en fonction de la température $(\mathrm{t})$ du lait écrémé $\left(\mathrm{Q}=51 . \mathrm{h}^{-1} ; \mathrm{v}=1,73 \times 10^{-2} \mathrm{~m} \cdot \mathrm{s}^{-1}\right.$; $\operatorname{Re} \simeq 190) ; \Delta t=10^{\circ} \mathrm{C} ;$ durée $=10 \mathrm{~min} ;$ préchauffeur de $4 \mathrm{~m}$.

Fouling mass $(m)$ on $304 \mathrm{~L}$ stainless steel versus temperature $(t)$ for skim-milk $\left(Q=51 . \mathrm{h}^{-1} ; v=1,73 \times 10^{-2} \mathrm{~m} \cdot \mathrm{s}^{-1} ; \mathrm{Re} \simeq 190\right) ; \Delta t=10^{\circ} \mathrm{C} ;$ contact time $=10 \mathrm{~min} ;$ preheater with $4 \mathrm{~m}$ length.

l'épaisseur du canal d'écoulement (e =0,2 $\mathrm{mm}$ ) la présence de dépôt est indiquée par une irisation de la surface mais sa masse n'est plus accessible par pesée, même lorsque l'essai dure $35 \mathrm{~min}$.

\section{DISCUSSION}

L'étude de l'encrassement d'un échangeur de chaleur par un fluide biologique comme le lait met en évidence une répartition inégale du dépôt suivant la position dans l'appareil (Lalande et al., 1981 ; Desmares, 1982). En effet, l'encrassement en un endroit donné est fonction des conditions hydrodynamiques ( $\mathrm{Re}, \mathrm{v} .$. ) et thermiques $(t, \Delta t)$ locales et du profil thermique dans la partie amont de l'échangeur. Ce dernier facteur influe sur le pouvoir encrassant au même titre qu'un certain nombre d'autres (âge du lait, propreté, conservation au froid...) (Burton, 1966) qui sortent du cadre de notre étude.

L'étude locale de l'encrassement nécessite de pouvoir faire varier l'ensemble de ces facteurs de façon indépendante. 


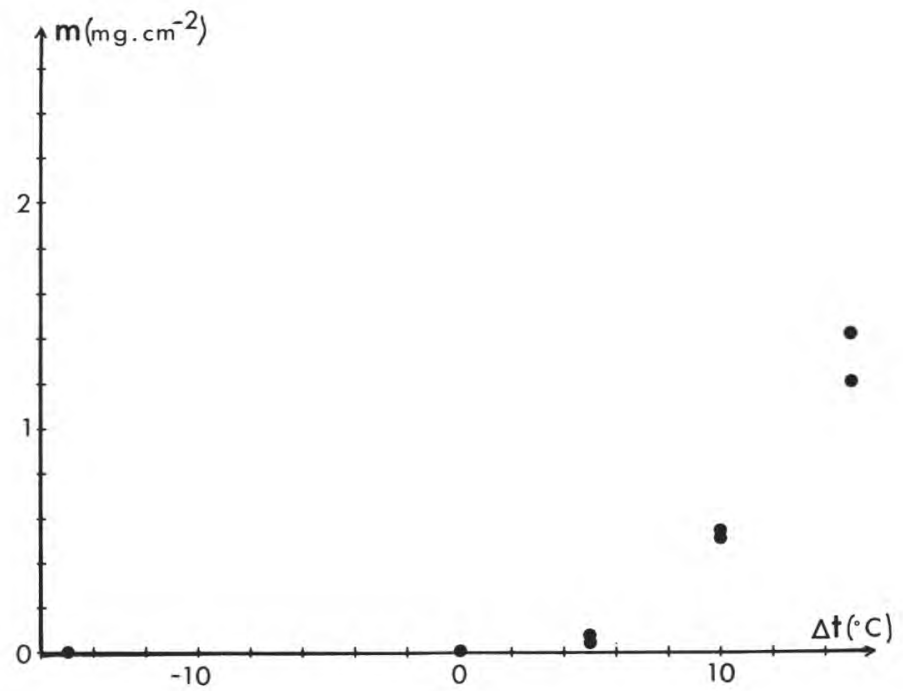

fig. 8

Evolution de la masse de dépôt $(\mathrm{m})$ sur acier inoxydable $18 \mathrm{Cr}-10 \mathrm{Ni}$ en fonction de l'écart de température $(\Delta t)$ entre le fluide chauffant ou refroidissant et le lait écrémé en régime laminaire $\left(\mathrm{Q}=51 \mathrm{~h}^{-1} ; \mathrm{v}=1,73 \times 10^{-2}\right.$ m.s. $\left.{ }^{1} ; \mathrm{Re} \simeq 190\right)$; $\mathrm{t}=80^{\circ} \mathrm{C}$; durée $=10 \mathrm{~min} ;$ préchauffeur de $4 \mathrm{~m}$.

Fouling mass $(m)$ on $304 L$ stainless steel versus temperature difference $(\Delta t)$ between heating or cooling fluid and skim-milk in laminar flow $(Q=5$ l.h-1 ; $\left.v=1,73 \times 10^{-2} \mathrm{~m} \cdot \mathrm{s}^{-1} ; R e \simeq 190\right) ; t=80^{\circ} \mathrm{C} ;$ contact time $=10 \mathrm{~min} ;$ preheater with $4 \mathrm{~m}$ length.

\subsection{Aspects thermiques}

Assurer le préchauffage du fluide encrassant par circulation dans un tube immergé dans un bain-marie à température constante est très simple dans la réalisation. Afin d'avoir une montée en température aussi rapide que possible et en particulier un temps de séjour à haute température aussi court que possible, il faut limiter la longueur du préchauffeur à $4 \mathrm{~m}$ ou moins (fig. 2), ce qui représente, au débit de $101 . \mathrm{h}^{-1}$, un temps de séjour maximal de 55 secondes. Ce dernier est du même ordre de grandeur que celui mesuré (37 secondes) dans notre prototype travaillant à $1 \mathrm{~m}^{3} \cdot \mathrm{h}^{-1}$ avec un taux de récupération de $93 \%$ (Desmares, 1982).

Pour se rapprocher encore plus du profil quasi-linéaire rencontré dans les échangeurs de chaleur industriels à contre-courant, en particulier aux faibles débits qui nous intéressent (fig. 3), nous nous proposons de réduire à $2 \mathrm{~m}$ la longueur du tube. Nous éviterons ainsi, dans les essais à venir, un temps de séjour inutilement long du produit aux températures voisines de la température de traitement 
choisie. Le profil thermique dans le préchauffeur sera légèrement modifié puisque pour obtenir une même température de sortie qu'avec $4 \mathrm{~m}$, nous devrons remonter la température du bain-marie. En outre, le temps de préchauffage étant réduit, nous devons nous attendre à un encrassement plus fort dans la maquette. Des essais préliminaires avec du lactosérum ont confirmé cette prévision : après 30 min d'encrassement $\left(\mathrm{Q}=101 . \mathrm{h}^{-1} ; \mathrm{t}=80^{\circ} \mathrm{C} ; \Delta \mathrm{t}=+10^{\circ} \mathrm{C}\right) \mathrm{la}$ masse de dépôt est de 200 et $950 \mu \mathrm{g} . \mathrm{cm}^{-2}$ pour respectivement 4 et $2 \mathrm{~m}$ de tube.

Par ailleurs, les possibilités de préchauffage sont limitées par le débit (fig. 3). Ainsi, pour une température de bain marie de $98^{\circ} \mathrm{C}$ la température du lait à la sortie ne peut dépasser, par exemple, $64^{\circ} \mathrm{C}$, pour un débit de $1001 . h^{-1}$. Aussi, pour effectuer des essais à débits élevés (étude à valeurs élevées de $\operatorname{Re}$ ou v), nous devons envisager l'utilisation d'un préchauffeur à plaques comportant une zone de récupération à contre courant (profil thermique linéaire ; limitation de la puissancec thermique à fournir), modulaire (profil thermique identique pour différents débits).

Les variations de température dans la maquette étant faibles (inférieures à $1^{\circ} \mathrm{C}$ ) on peut la considérer comme représentative d'une surface d'aire réduite d'un échangeur à plaques. Associée au préchauffeur décrit et discuté ci-dessus, elle constitue un ensemble expérimental de laboratoire permettant de simuler convenablement les conditions thermiques existant en un point quelconque d'un échangeur industriel.

\subsection{Aspects hydrodynamiques}

La limitation à 1 bar de l'étendue de mesure de notre capteur de pression et dans certains cas la limitation à $2001 . h^{-1}$ de notre pompe ne permettent pas toujours de mettre en évidence les changements de régime d'écoulement. Ainsi, pour la maquette à e $=0,1$ et $0,4 \mathrm{~mm}$ les valeurs expérimentales de Re n'ont pu dépasser 400 et 6500 respectivement.

De plus, aux faibles valeurs de pression, l'erreur faite sur la mesure du zéro du capteur entraîne une grande incertitude sur Da, illustrée pour la maquette à e $=1,47 \mathrm{~mm}$ pour les faibles valeurs de Re. Cette incertitude conduit, suivant les essais, à des courbes très différentes (fig. 4, courbes a, b, c, d) dont certaines n'ont assurément aucune signification physique. Pour le prototype, ce manque de précision du capteur à des faibles valeurs de différences de pression, entraîne également des difficultés pour explorer les régimes correspondant à de faibles débits ; un capteur couvrant une plage plus faible de différences de pressions aurait sensiblement amélioré l'expérimentation.

Pour la maquette à e variant de 0,1 à $0,4 \mathrm{~mm}$ l'exposant $\mathrm{m}$ de Re dans la relation (10) est voisin de -1 (tab. 3), valeur donnée par 
Fortier (1975) et Rohsenow et Hartnett (1973 b) pour le régime laminaire dans un canal de section rectangulaire.

La valeur de $\mathbf{k}$ donnée par ces mêmes auteurs est de 96 pour la géométrie des canaux étudiés et s'éloigne sensiblement de celles trouvées lors de nos expérimentations (50 à 74). Il en est de même pour les valeurs critiques de $\mathrm{Re}$ à la fin du régime laminaire qui varient de 270 à 650 dans nos essais contre 2400 à 2800 suivant Rohsenow et Hartnett (1973 b).

Quelques hypothèses peuvent être avancées pour expliquer ces différences :

- les épaisseurs de canaux concernées sont très faibles (inférieures à $0,4 \mathrm{~mm}$ ) et sans doute hors du domaine couvert par les données bibliographiques, bien que celles-ci ne précisent pas ce point ;

- les forces de tension superficielle (caractérisées par le nombre de Weber : We $=\frac{\rho 1 \mathrm{v}}{\gamma}$, avec $\gamma=$ tension superficielle) ont été négligées ;

- en raison de la faible longueur du canal de passage, le régime n'est pas établi dans la totalité de la maquette ;

- la géométrie des tubulures d'admission de la maquette peut jouer le rôle de promoteur de turbulence.

Nous n'avons pu, pour l'instant, recueillir d'éléments privilégiant l'une ou l'autre de ces hypothèses.

Dans le prototype, seuls la fin du régime de transition et le début du régime turbulent sont explorés. En effet, d'après Edwards et al. (1974) et Price et Fattah (1978), dans un échangeur à plaques type APV junior Paraflow, le régime laminaire se situe à des valeurs de Re inférieures à 20. D'après ces mêmes auteurs, le régime de transition se situe à des valeurs de Re comprises entre 10 et 400 . Corrieu et al. (1978) ont trouvé sur un échangeur identique au nôtre, une zone de transition allant jusqu'à $R e$ voisin de 500 . La valeur de 970 trouvée dans nos essais est donc légèrement supérieure à ces valeurs.

En régime turbulent, le prototype a un comportement proche de celui prévu par le constructeur : $\mathrm{Da}=9$ Re-0,20 et exactement identique à celui trouvé par Price et Fattah (1978) pour l'échangeur APV junior paraflow : $\mathrm{Da}=7 \mathrm{Re}-0,20$.

L'ensemble de ces résultats montre une absence de similitude entre prototype et maquette qui peut probablement s'expliquer par la différence de géométrie des canaux d'écoulement : dans le prototype les plaques sont ondulées et ponctuellement en contact avec la plaque voisine ; dans la maquette la plaque est plane. Par contre nous n'avons pas d'hypothèse pour expliquer l'absence de similitude, pour la maquette, entre les canaux d'épaisseurs différentes. 
La figure 5 indique, pour de l'eau à $19^{\circ} \mathrm{C}$, les couples $\mathrm{Re}, \mathrm{v}$ que l'on peut couvrir avec la maquette. Un débit de $3001 . h^{-1}$ permet de se placer dans la zone de travail d'un échangeur industriel ( $\mathrm{v}=1 \mathrm{~m} . \mathrm{s}^{-1}$; $\mathrm{Re}=7000$ ) avec une maquette de $\mathrm{e}=4 \mathrm{~mm}$. En faisant varier cette épaisseur, on peut à ce même débit, couvrir une gamme de vitesse de 1 à $10 \mathrm{~m} \cdot \mathrm{s}^{-1}$ à $\mathrm{Re}$ constant voisin de 7000 . En jouant à la fois sur le débit et l'épaisseur du canal, on peut aussi obtenir des nombres de Reynolds variables de 1500 à 8000 à vitesse constante voisine de $1 \mathrm{~m} \cdot \mathrm{s}^{-1}$. Globalement donc, la maquette, avec son canal de faible largeur $(20 \mathrm{~mm})$, permet d'effectuer des études en diminuant considérablement, par rapport au propotype, les quantités de produit mises en œuvre. Enfin, la maquette d'épaisseur de canal 0,1 à $0,4 \mathrm{~mm}$ permet de couvrir le domaine laminaire pour des débits inférieurs à environ $101 . h^{-1}$ et d'aborder le domaine de transition pour les débits supérieurs.

Sur la figure 5 , les couples de valeurs expérimentales, Re critique$\mathrm{v}$, sont indépendants du fluide et de la température. Par contre, les droites d'isoépaisseur et les courbes d'isodébit peuvent être tracées pour des fluides autres que l'eau, sous réserve de prendre en compte dans les relations (8) et (9) les valeurs de leurs caractéristiques physiques $(\rho, n)$ à la température de traitement thermique.

Il est intéressant de noter que Meyer et al. (1981) ont utilisé une maquette très semblable à la nôtre (canal d'épaisseur 1,52 $\mathrm{mm}$ et de largeur $14 \mathrm{~mm}$ ) qui a d'ailleurs fait l'objet d'un brevet (De Palma et Baier, 1979). Cet appareil a permis d'aborder l'étude de l'encrassement des échangeurs de chaleur de l'industrie nucléaire, par les substances biologiques de différentes eaux. Lors de leur simulation, ils ont utilisé un débit de 19,51.h-1 sans préciser le régime d'écoulement.

\subsection{Encrassement}

Nos essais d'encrassement étaient principalement destinés à éprouver la validité de la maquette. De ce point de vue, malgré leur dispersion, nos résultats confirment nettement l'influence bien connue des paramètres classiques que sont, en particulier, la durée de contact, la température du fluide encrassant ( $t)$ et l'écart de température $(\Delta t)$ (Gynning et al., 1958 ; Thom, 1970 ; Lalande et al., 1981). La structure des dépôts formés est en accord avec celle décrite par Thom (1975) qui observe en particulier des alvéoles aux faibles vitesses de circulation du fluide et lorsque l'écart de température avec le fluide chauffant est suffisamment élevé. De plus, dans la maquette, l'aspect des dépôts est semblable à celui observé dans le prototype. Il est vraisemblable, ainsi que le suppose Thom (1975), que les alvéoles soient consécutives à la formation des bulles de gaz libéré par l'élévation de température du produit. En effet, ni le prototype, ni la maquette ne possèdent de contre pression pour éviter ce phénomène. Les conséquences de ce dernier s'estompent avec l'augmentation de la vitesse de circulation du produit. 
Ces quelques résultats montrent que notre maquette permet de reproduire les conditions de l'encrassement et d'obtenir des résultats parfaitement conformes aux données actuellement connues concernant l'encrassement macroscopique.

L'étude des premières phases de l'encrassement, inaccessibles par évaluation de la masse de dépôt, nécessite la mise en œuvre de techniques fines d'analyse et de mesure des caractéristiques de surface. La planéité et les petites dimensions de la plaque d'étude permettent d'envisager la mesure, en temps différé, des caractéristiques optiques des films minces d'encrassement. Ainsi, l'éllipsométrie a permis à Fort (1983) d'étudier la cinétique d'adsorption lors du contact entre une solution de caséinate de sodium et de l'acier inoxydable ou de l'argent. En outre, ces plaques se prêtent bien à la spectroscopie d'électrons rétrodiffusés Auger (Fort, 1983), et à la spectroscopie de photo-électrons X (XPS ou ESCA) (Ratner et al., 1981) en vue de l'identification des espèces adsorbées et de leur liaison avec la surface étudiée. L'évaluation des caractéristiques énergétiques (énergie de surface et tension critique de surface) est également permise par l'intermédiaire de la mesure d'angle de contact (Zisman, 1964 ; Kaelble et Moacanin, 1977 ; De Palma et Baier, 1978 ; Baszkin et Lyman, 1980 ; Meyer et al. 1981). Enfin, des mesures de nature électrochimique (potentiel, courant, impédance) sont envisageables en temps réel (Beljelti et al. 1980 ; Ivarsson et al., 1981), après implantation d'une électrode de référence et d'une contre électrode de platine, sur la plaque qui présente la particularité avantageuse d'être isolée électriquement du reste du dispositif par les joints en polytétrafluoréthylène.

\section{CONCLUSION}

La maquette et son environnement semblent réunir les conditions nécessaires à l'étude des premières phases de l'encrassement au cours des traitements thermiques de liquides alimentaires. Ils assurent la maîtrise et la connaissance du profil de montée en température du produit dans le préchauffeur et du niveau de température à l'entrée de la maquette. L'existence de conditions thermiques et hydrodynamiques homogènes et quasi-constantes dans le temps entre le fluide et la surface est obtenue par les petites dimensions des échantillons dont la faible largeur permet en outre de mettre en œuvre des quantités réduites de liquides. Ces dimensions et la planéité de la surface sont en outre indispensables à l'utilisation de techniques fines d'étude des propriétés physicochimiques de surface, certaines d'entre elles pouvant d'ailleurs être mises en œuvre en temps réel.

Les paramètres hydrodynamiques (nombre de Reynolds et vitesse moyenne de passage) et thermiques (niveau et écart de température) peuvent être fixés indépendamment les uns des autres et varier 
dans de larges plages. Cependant, les résultats présentés n'ont pas permis d'identifier complètement les régimes d'écoulement dans la maquette. Une expérimentation approfondie, s'appuyant sur des moyens de mesure plus appropriés pourrait permettre d'apporter les précisions qui manquent encore.

On peut cependant envisager l'étude de l'influence :

- de la composition du produit traité (incluant le passé thermique);

- des conditions d'écoulement (Re et v) ;

- des conditions thermiques locales ( $t$ et $\Delta t$ ) ;

- de la nature et de l'état de surface du matériau solide sur l'encrassement et en particulier ses premières phases.

\section{Remerciements}

Nous tenous à remercier notre collègue Marc Lalande (Laboratoire de Génie Industriel, I.N.R.A. Lille) pour les critiques utiles et constructives que la lecture du manuscrit de cet article lui a inspirées.

\section{Bibliographie}

Amariglio (S.), Serres (L.), Petranxienne (D.) (1973). - Contrôle de la qualité des produits laitiers. 1. Analyse physique et chimique. Direction des services vétérinaires. Laboratoire central de l'inspection du lait.

Aschaffenburg (R.) and DRewRy (J.) (1959). - New procedure for the routine determination of the various non casein proteins of milk. Int. Dairy Cong. London, 3, 1631-1637.

BAIER (R. E.) (1981). - Modification of surfaces to reduce fouling and or improved cleaning. Fundamentals and applications of surface phenomena associated with fouling and cleaning in food processing. Proceedings Tylösand, 168-169.

BASZkin (A.) and LyMAN (D. J.) (1980). - The interaction of plasma protein with polymers. I. Relationship between polymer surface energy and protein adsorption/desorption, J. Biomed. Mat. Res., 14, 393-403.

Beljelti (S.), Ben Bachir (A.), Dumas (J. P.), El Adioui (S.), Jouanneau (A.) et Petit (M. C.) (1980). - Etude des transformations de quelques protéines au contact du nickel. Materials Chemistry (5), 7-18.

Bertsch (A.) (1981). - Propriétés physiques du lait et des crèmes au-dessus de $100^{\circ}$ C. Thèse Docteur-Ingénieur, E.N.S.I.A.A. Massy.

Bixвy (D. G.) (1974). - Fouling of heat exchange surfaces by skim milk. Dissertation abstract international, 35, 1287-1288.

BURTon (H.) (1961). - A laboratory method for the investigation of milk deposits on heat exchange surfaces. J. Dairy Res., 28, 255-263.

BURToN (H.) (1965). - A method for studying the factors in milk which influence the deposition of milk solids on a heated surface. J. Dairy Res., 32, 65-78.

BuRTon (H.) (1966). - The effect of forewarming on the formation of deposits from separated milk on a heated wire. 17 th Int. Dairy Cong., Munich, $B, 607$. 
Burton (H.) (1968). - Deposits from whole milk in heat treatment plant: a review and discussion. J. Dairy Res., 35, 317-330.

CABARAT (I.) (1980). - Viscosité et masse volumique de rétentats d'ultrafiltration. Mémoire de fin d'études, D.E.A. Sciences Alimentaires, option lait, Rennes.

CoRrieu (G.) et LAlande (M.) (1978). - Pasteurisation du lait sur un échangeur de chaleur à plaques "Vicarb ». Rapport de fin d'étude de pré-développement, I.N.R.A., Ministère de l'Industrie.

Cultoli (J.), Bon (J.P.) et Maubois (J.L.) (1974). - Etude de la viscosité des " rétentats " et des " préfromages " obtenus après traitement du lait par ultrafiltration sur membrane. Lait, 54, 481-500.

De Palma (V. A.) and BAIER (R. E.) (1978). - Proceedings of the ocean thermal energy conversion biofouling and corrosion symposium. Seattle.

De Palma (V.A.) and Bater (R. E.) (1979). - Flow cell and method for continuously monitoring deposits on flow surfaces. U.S. Patent $n^{\circ} 4,175,233$.

Desmares (A.) (1982). - Encrassement d'un échangeur à plaques lors de traitements thermiques du lait et de rétentats d'ultrafiltration. Aspects thermiques et hydrodynamiques. Mémoire de fin d'études, S.P.I.L.A.B. Douai.

Edwards (M. F.), Changal Vaie (A. A.) and Parrott (D. I.) (1974). - Heat transfer and pressure drop characteristics of a plate heat exchanger using Newtonian and non Newtonian liquids, Chemical Engineer., (285), 286-288 and 293.

FERNANDEZ-MARTIN (F.) (1972). - Influence of temperature and composition on some physical properties of milk and milk concentrates. I. Heat capacity. J. Dairy Res., 39, 65-73, II. Viscosity. J. Dairy Res., 39, 75-82.

Fernandez-Martin (F.) and Montes (F.) (1972). - Influence of temperature and composition on some physical properties of milk and milk concentrates. III. Thermal conductivity. Milchwiss., 27 (12), 772-776.

FORT (J.) (1983) . - Contribution à l'étude d'interactions caséinate de sodiumacier inoxydable austénitique ou argent ; mise en évidence et développements de films adsorbés à la surface des matériaux. Thèse de Docteur-Ingénieur, Toulouse.

Fortier (A.) (1975). - Mécanique des fluides et transferts de chaleur et de masse par convection. Masson.

(1981). - Fundamentals and applications of surface phenomena associated with fouling and cleaning in food processing. Proceedings Tylösand.

GoRdon (K. P.), HANKINSON (D. J.) and CARVER (C. E.) (1968). - Deposition of milk on heated surfaces. J. Dairy Sci., 51, 520-526.

Gynning (K.), Thome (K. E.) and Samuelsson (E. G.). (1958). - The burning of milk in milk pasteurizers. Milchwiss., 13, 62-70,

Hegg (P. O.) and LARsson (K.) (1981). - Ellipsometry studies of adsorbed lipids and milk proteins on metal surfaces. Fundamentals and applications of surface phenomena associated with fouling and cleaning in food processing. Proceedings Tylösand, 250-255.

Idel'cik (I. E.) (1969). - Memento des pertes de charges. Eyrolles, Paris, 96, 122, 199.

IVARSSON (B.), JONSSON (U.) and LUNDSTROM (I.) (1981). - Ellipsometrical and electrochemical studies of adsorption at solid-liquid interfaces. Fundamentals and applications of surface phenomena associated with fouling and cleaning in food processing. Proceedings Tylösand, 269-278.

Kaelble (D. H.) and Moacanin (J.) (1977). - A surface energy analysis of bioadhesion. Polymer, 18 (5), 475-482.

Kessler (H. G.) (1981). - Food engineering and dairy technology. Kessler Freising. 
Lalande (M.), Corrieu (G.), Daufin (G.) et Gallot-Lavallée (T.) (1981). - Etude des cinétiques d'encrassement et de nettoyage d'un pasteurisateur de lait à plaques. C.R. fin d'études D.G.R.S.T., 79.7.0375,

LING (A. C.) and LuND (D. B.) (1978). - Apparatus for studying fouling of heated surfaces by biological fluids. J. Food Sci., 43 (2), 390-393, 403.

LUND (D. B.) and BIXBY (D.) (1975). - Fouling of heat exchange surfaces by milk. Proc. Biochem., 10 (9), 52-55.

LUND (D. B.) and SANDU (C.) (1981). - State-of-the-art of fouling: heat transfert surfaces. Fundamentals and applications of surface phenomena associated with fouling and cleaning in food processing. Proceedings Tylösand, 27-56.

LYSTER (R. L. J.) (1965). - The composition of milk deposits in an U.H.T. plant. J. Dairy Res., 32 (1), 203-208.

Meyer (A. E.), Baier (R. E.) and King (R. W.) (1981). - Mitigation of biofouling using coatings. Calspan report $\mathrm{n}^{\circ}$ 6792-M-4.

OGG (C. L.) (1960), - Determination of nitrogen by the micro-Kjeldahl method. J. A.O.A.C., 43, 689-693

PRICE (A. F.) and FATTAH (A. F. M. A.) (1978). - Hydrodynamic characteristics of a plate heat exchanger channel. Trans I Chem E, 56, 217-227.

Ratner (B. D.), HorbetT (T. A.), Shuttleworth (D.) and Thomas (H. R.) (1981). Analysis of the organization of protein films on solid surfaces by E.S.C.A. J. Colloid Interf. Sci., 83 (2), 630-642.

RohSEnOw and HaRTNETT (1973 a). - Handbook of heat transfer. Mc Graw-Hill, 7.212 and 7.123 .

RoHSENow and HARTNETT (1973 b). - Handbook of heat transfer Mc Graw-Hill, 7.112 and 7.116 .

Rota (A.) and Bottazzi (V.) (1978). - Progress in the technique of milk pasteurization. 20th Int. Dairy Congress, 678.

Tном (R.) (1970). - Ueber die Struktur des Milchansatzes in Plattenerhitzern. Milchwiss., 25 (9), 519-528.

Tном (R.) (1975). - Ueber die Bildung von Milchansatz in Plattenerhitzern. Milchwiss., 30 (2), 84-90.

Zisman (W. A.) (1964). - Advances in chemistry series. Am. Chem. Soc., R. Gould, $43,1-51$. 